\title{
The protective effect of Gingko biloba in a rat model of ovarian ischemia/reperfusion injury: Improvement in histological and biochemical parameters
}

\author{
Nuri Yildirim ${ }^{1, A, D, F}$, Deniz Simsek ${ }^{1, C, D}$, Semir Kose ${ }^{2, C, E}$, Alkim Gulsah Sahingoz Yildirim ${ }^{3, B, E}$, \\ Cagri Guven ${ }^{1, C, E}$, Gurkan Yigitturk ${ }^{4, B, E}$, Oytun Erbas ${ }^{5, A, E, F}$ \\ ${ }^{1}$ Department of Obstetrics and Gynecology, Ege University, Izmir, Turkey \\ 2 Department of Obstetrics and Gynecology, Dokuz Eylul University, Izmir, Turkey \\ ${ }^{3}$ Department of Obstetrics and Gynecology, Tepecik Maternity and Research Hospital, Izmir, Turkey \\ ${ }^{4}$ Department of Histology and Embryology, Ege University, Izmir, Turkey \\ ${ }^{5}$ Department of Physiology, Bilim University, Istanbul, Turkey \\ A - research concept and design; $\mathrm{B}$ - collection and/or assembly of data; $\mathrm{C}$ - data analysis and interpretation; \\ $D$ - writing the article; $E$ - critical revision of the article; $F$ - final approval of the article
}

Address for correspondence

Nuri Yildirim

E-mail: nuri-yildirim@hotmail.com

\section{Funding sources}

None declared

\section{Conflict of interest}

None declared

Received on June 5, 2016

Reviewed on August 24, 2016

Accepted on February 9, 2017

DOI

10.17219/acem/68896

\section{Copyright}

Copyright by Author(s)

This is an article distributed under the terms of the

Creative Commons Attribution Non-Commercial License

(http://creativecommons.org/licenses/by-nc-nd/4.0/)

\begin{abstract}
Background. Ovarian torsion is one of the most common gynecological emergencies, which especially affects women of reproductive age.

Objectives. We aimed to evaluate the effect of Ginkgo biloba (GB) supplementation in ovarian ischemia/ reperfusion injury in an experimental torsion/de-torsion rat model.

Material and methods. This study was carried out in the Ege University Faculty of Medicine in Izmir, Turkey. Thirty mature female Sprague-Dawley albino rats were randomly divided into 5 groups: in Group 1 (control), the abdominal wall was only opened and closed; in the torsion group (Group 2), ischemia was induced for $3 \mathrm{~h}$, using atraumatic vascular clips to create a torsion model; in the torsion/GB group (Group 3), the rats were given $80 \mathrm{mg} / \mathrm{kg}$ (oral gavage) of GB 30 min before torsion was induced and the torsion model was formed; in the torsion/de-torsion group (Group 4), the rats underwent $3 \mathrm{~h}$ of ischemia and then the vascular clips were removed and reperfusion took place for $3 \mathrm{~h}$; in the torsion/de-torsion/GB group (Group 5), the rats underwent $3 \mathrm{~h}$ of ischemia followed by GB (oral gavage) 30 min prior to a 3 -h reperfusion period. Ovarian tissue damage was evaluated by a histopathological scoring system. Ovarian tissue malondialdehyde (MDA) and plasma pentraxin-3 were measured.
\end{abstract}

Results. In comparison with the sham group, both the torsion and torsion/de-torsion groups had significantly higher scores for follicular degeneration, vascular congestion, edema, hemorrhage, and leukocyte infiltration. Ginkgo bilobasignificantly decreased thesescores in both groups. Ovarian malondialdehyde and plasma pentraxin 3 were significantly higher both in the torsion and torsion/de-torsion groups compared with the sham group. Ginkgo biloba decreased these levels significantly both in the torsion/GB and torsion/de-torsion/GB groups.

Conclusions. Supplementing GB during a surgical procedure decreases ischemia/reperfusion injury to an ovary in an experimental rat model based on histopathological parameters, tissue malondialdehyde, and plasma pentraxin-3 levels.

Key words: malondialdehyde, ischemia/reperfusion injury, ovarian torsion, Ginkgo biloba, pentraxin-3 


\section{Introduction}

Ovarian torsion is defined as a partial or total rotation of the ovary, the fallopian tube, or both around its vascular axis. The compression of the ovarian vessel obstructs lymphatic and venous outflow first, and subsequently arterial inflow. The blockage of the lymphatic and venous systems leads to ovarian edema, resulting in enlargement of the ovary. Increased ovarian stromal pressure blocks the arterial blood supply, which leads to ovarian ischemia and results in necrosis of the ovarian tissue, infarction, and local hemorrhage. It is the $5^{\text {th }}$-leading cause of surgical emergencies with a prevalence of $2.7-3 \%{ }^{1}$ However, increased infertility treatment tends to raise the incidence rate iatrogenically. The incidence rate rises dramatically to $6 \%$ after ovarian stimulation and reaches as high as $16 \%$ in cases of ovarian hyperstimulation syndrome. ${ }^{2}$

The majority of ovarian torsion cases occur at reproductive age. ${ }^{3}$ The diagnosis is often challenging because of the non-specific signs, symptoms, and limited benefits of imaging techniques and laboratory markers. The classic presentation of ovarian torsion is acute abdominal pain along with nausea, vomiting, and positive peritoneal signs. Additionally, adnexal masses or the impairment of ovarian blood flow would provide support for a diagnosis. Nevertheless, suspicion of ovarian torsion should be kept in mind in order to reduce adverse outcomes, especially the loss of ovarian function. Immediate surgical intervention is necessary as soon as ovarian torsion is diagnosed. In the past, fear of thromboembolic phenomena or a suspected malignant mass led physicians to perform an oophorectomy. However, there are no reported cases of embolic incidents after de-torsion in the literature. Thus, the current recommendation is to salvage the twisted ovary with de-torsion even if the tissues are cyanotic. ${ }^{4}$

De-torsion of the twisted ovary replenishes the blood supply and initiates the recovery process. Paradoxically, the return of blood flow can also result in additional ovarian damage and complications, referred to as reperfusion injury. Ischemia injury occurs because of an imbalance between energy requirements and production. ${ }^{5}$ Aerobic energy cannot be utilized in an ischemic patient; the required energy is obtained through the anaerobic pathway. Remnants of this process can damage the cell. These processes are reversible only if reperfusion is instituted. However, reperfusion can be also hazardous because of neutrophil infiltration and excessive production of reactive oxygen species (ROS), called ischemia/reperfusion (I/R) injury. Membrane lipids are the most sensitive structures affected by ROS. Several substances are produced during the lipid oxidation process. Malondialdehyde is one of the most important end-products of lipid peroxidation and is used to indicate the level of oxidative damage. Pentraxin-3 (PTX3) is an acute-phase reactant produced predominantly by macrophages, dendritic cells, and endothelial cells. It functions as a central immune mediator, which is a novel marker for $\mathrm{I} / \mathrm{R}$ injury, especially in myocardial cells. $^{6,7}$

Ovarian viability and function is related not only to the period of ischemia, but also to reperfusion injury. Recently, many agents like edaravone, oxytocin, and octreotide have been reported to improve the side effects of I/R injury in animal models because of the their anti-inflammatory and antioxidant effects. ${ }^{8-10}$

Ginkgo biloba is one of the world's oldest living tree species. It contains dozens of active substances, including flavonoids, terpene lactones, ginkgo flavone glycosides, and bilobetin. Several investigators have used GB to treat dementia, tinnitus, cerebral insufficiency, sexual dysfunction, vertigo, vitiligo, macular degeneration, and peripheral vascular disease. The mechanism has not yet been defined exactly, but it has neuromodulator effects in the central and peripheral nervous system: it is an antioxidant and a vasodilator. As an antioxidant, GB reduces superoxide release in polymorphonuclear cells, acts as a scavenger for free radicals, and also acts as a mediator of lipid peroxidation and cell damage. ${ }^{11-13}$ Recently, GB extracts were screened by ultra-high performance liquid chromatography. Sixtyone compounds were identified, 25 of which showed considerable radical scavenging capacity. This means that GB could have an important protector role not only in ischemic injury, but also in ischemic/reperfusion injury. ${ }^{14}$

To date, there have been several studies investigating the beneficial role of GB on various systems of the body; however, there is limited data on the effect of on ovarian ischemia/reperfusion injury. Therefore, the aim of the present study was to investigate the effect of GB on I/R injury in a rat ovary torsion/de-torsion model using a histopathological score, ovarian tissue MDA, and plasma PTX3 as tissue and systemic I/R injury markers.

\section{Material and methods}

\section{Animals}

We used 30 mature (12-week-old) female Sprague-Dawley albino rats that weighed 200-220 g each. The animals were fed ad libitum and housed in pairs in steel cages in a temperature-controlled environment $\left(22 \pm 2^{\circ} \mathrm{C}\right)$ with 12-h light/dark cycles.

The local Animal Ethics Committee approved the experimental procedures employed in the present study. All experiments were carried out according to the Guide for the Care and Use of Laboratory Animals, as confirmed by the National Institute of Health (USA).

The estrus stage of each rat was determined by taking a vaginal smear at an interval of 6-12 h. Cells types in the smear were subsequently examined under a microscope according to the Papanicolaou stain procedure. Only the 30 rats, which were confirmed to be in the estrus stage by the smear, were included in the experiment. 


\section{Experimental protocol}

Rats were anesthetized by an intraperitoneal injection of a combination of $50 \mathrm{mg} / \mathrm{kg}$ ketamine hydrochloride and $7 \mathrm{mg} / \mathrm{kg}$ xylazine hydrochloride (Alfazyne; Alfasan International BV, Woerden, Holland). After shaving and disinfection, a lower midline laparotomy was performed by making a 2-cm longitudinal incision, and the uterine horns and adnexa were located.

In Group 1 ( $\mathrm{n}=6$, sham-operated control group), only a laparotomy was performed and then closed with $3 / 0$ silk sutures (12). In Group 2 ( $n=6$, torsion group), ischemia was induced for $3 \mathrm{~h}$ by applying atraumatic vascular clips to the vascular pedicle $1 \mathrm{~cm}$ above and below the ovary. In Group 3 ( $n=6$, torsion and GB group), torsion was induced $30 \mathrm{~min}$ after the administration (oral gavage) of $80 \mathrm{mg} / \mathrm{kg}$ of $\mathrm{GB}$ (Tebokan, Abdi Ibrahim, Turkey). In Group 4 ( $n=6$ torsion/ de-torsion group), $3 \mathrm{~h}$ of ischemia and $3 \mathrm{~h}$ of reperfusion were performed. In Group 5 ( $n=6$, torsion/de-torsion and GB group), torsion was induced for $3 \mathrm{~h}$, followed by the administration of $80 \mathrm{mg} / \mathrm{kg}$ of GB (oral gavage) $30 \mathrm{~min}$ prior to de-torsion/reperfusion, which lasted $3 \mathrm{~h}$. Tablets containing 80 mg GB (Tebokan, Abdi Ibrahim, Turkey) were crushed and suspended in tap water to yield a concentration of $10 \mathrm{mg} / \mathrm{mL}$. According to the weight of each rat, the suspended drug solution was topped up to $4 \mathrm{~mL}$ with tap water. The medications were given via orogastric tubes. Immediately after the reperfusion period, both ovaries were excised for histological and biochemical evaluation.

\section{Histopathological examination}

The specimens were fixed in $10 \%$ buffered formalin for $48 \mathrm{~h}$, cleared in xylene, and embedded in paraffin. Tissue sections of 4- $\mu \mathrm{m}$ were stained with hematoxylin and eosin for morphologic analysis. All sections were photographed with an Olympus C-5050 digital camera located on an Olympus BX51 microscope (Olympus Corp., Tokyo, Japan). All primordial and developing follicles were examined histologically.

Follicular degeneration, vascular congestion, hemorrhaging, edema, and infiltration by inflammatory cells were scored from 0 to 3 according to the severity of injury, with 0 indicating no pathological findings and 1, 2, and 3 representing pathological findings of $<33 \%, 33-66 \%$, and $>66 \%$ of the ovarian section, respectively. ${ }^{15}$

\section{The measurement of tissue lipid peroxidation}

Ovarian tissues were homogenized in $150 \mathrm{mM}$ of ice-cold $\mathrm{KCl}$ and centrifuged at $5000 \mathrm{~g}$ for $10 \mathrm{~min}$. The supernatants were analyzed for lipid peroxidation. Lipid peroxidation was evaluated in each tissue sample by measuring the malondialdehyde level as thiobarbituric acid-reactive substances (TBARS). Briefly, trichloroacetic acid, TBARS reagent, and the tissue samples were mixed and incubated at $100^{\circ} \mathrm{C}$ for $60 \mathrm{~min}$. After cooling, the samples were centrifuged at $3000 \mathrm{rpm}$ for $20 \mathrm{~min}$ and the absorbance of the supernatant was read at $535 \mathrm{~nm}$. The MDA level was calculated from a standard calibration curve using tetraethoxypropane and expressed as $\mathrm{nmol} / \mu \mathrm{g}$ of protein. ${ }^{16}$

\section{Measurement of the tissue protein levels}

The total protein levels of the tissue samples were analyzed according to Bradford's method using bovine serum albumin as a standard. ${ }^{17}$

\section{Evaluation of plasma pentraxin-3 levels}

Plasma pentraxin-3 (PTX3) levels were assessed in each $100-\mu \mathrm{L}$ sample by the standard ELISA method at $450 \mathrm{~nm}$ using a PTX3 kit (USCN Life Science Inc., Wuhan, China). PTX3 levels were determined in duplicate according to the manufacturer's guide. The detection range for PTX3 assay was $0.078-5 \mathrm{ng} / \mathrm{mL}$.

\section{Statistical analysis}

Non-parametric variables were analyzed using the Kruskal-Wallis test. Parametric variables were evaluated with one-way ANOVA, followed by Tukey's HSD test with $\mathrm{p}<0.05$ accepted as statistically significant. SPSS v. 15.0 for Windows was used for analysis.

\section{Results}

The histopathological findings are shown in Fig. 1. The ovaries of the torsion group (Group 2) were compared with Group 1 according to the histopathological findings. All of the histopathological parameters were significantly higher in Group $2(\mathrm{p}<0.01$ and $\mathrm{p}<0.0001)$. In the torsion/GB group (Group 3), all these parameters except edema were significantly decreased $(\mathrm{p}<0.05)$ compared with Group 2. Decreased edema was detected in Group 3, but this difference did not reach statistically significant levels ( $\mathrm{p}>0.05)$. Comparison of the torsion/ de-torsion group (Group 4) and the torsion/de-torsion + GB group (Group 5) revealed that all histopathological parameters were significantly lower in Group 5 ( $\mathrm{p}<0.05, \mathrm{p}<0.01)$. The histopathological scores are listed in Table 1 and Fig. 2.

Tissue MDA and plasma PTX3 were evaluated as cell injury markers. Group 3 had lower values than Group 2 and these differences were statistically significant $(\mathrm{p}<0.05)$. Comparing Groups 4 and 5, Group 4 had higher values than Group 5 and this difference was statistically significant $(\mathrm{p}<0.001)$. Tissue MDA and plasma PTX3 are shown in Table 2 and Fig. 3. 


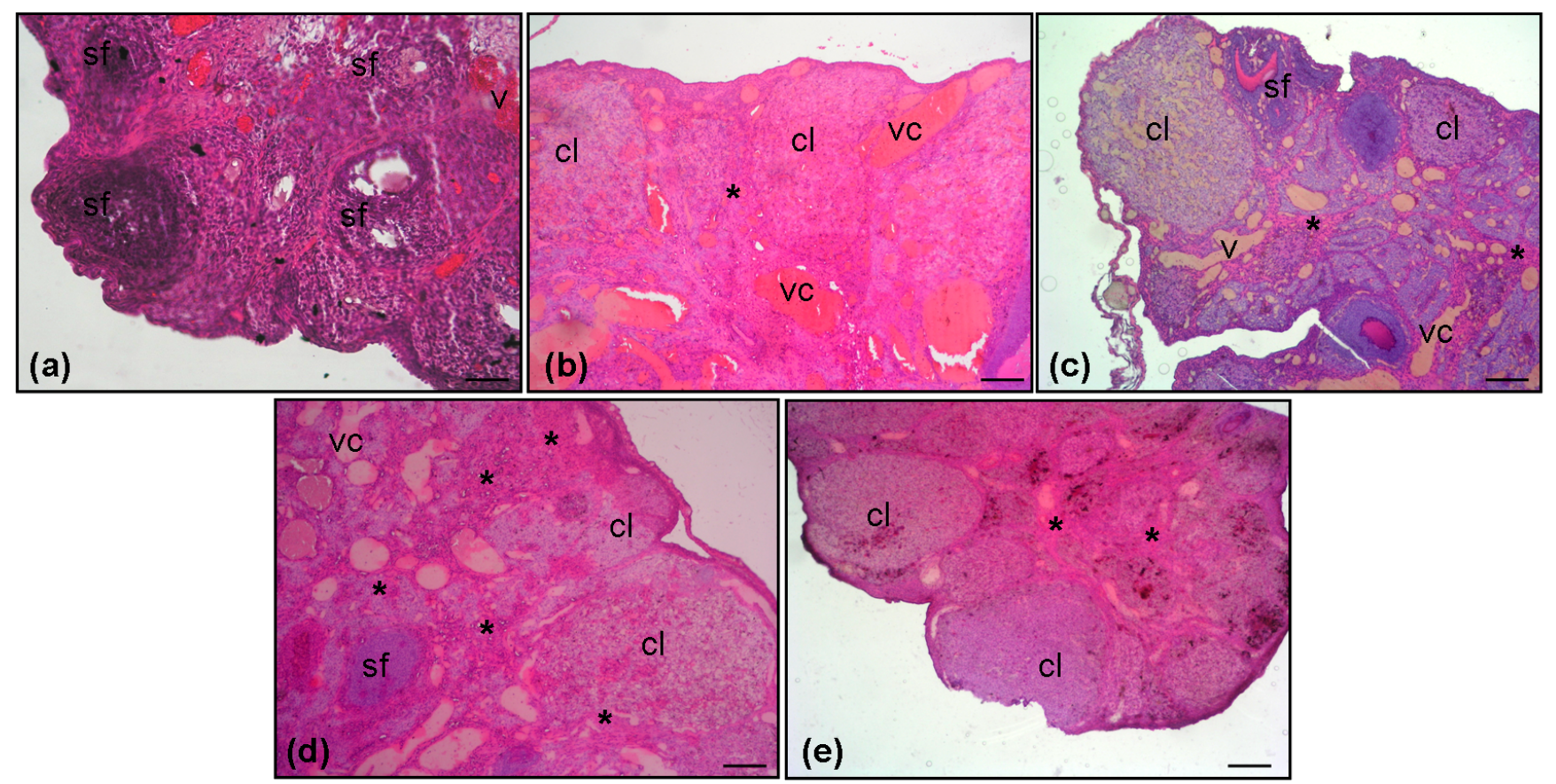

Fig. 1. Ovarian tissue sections of rats in Groups 1-5 are represented in pictures A-E, respectively. Hematoxylin and eosin staining was performed. Scale bars represent $250 \mu \mathrm{m}$

a - no pathological changes were detected in the sham group. (v) vessel, (sf) secondary follicle; b- edema (*), vascular congestion (vc) in the 3-h torsion group; $\mathrm{c}$ - decreased edema and vascular congestion in the torsion + GB group; $\mathrm{d}$ - vascular congestion (vc), edema $(*)$ in the torsion/detorsion group; e- decreased edema ${ }^{*}$ ) in the torsion/de-torsion + GB group.

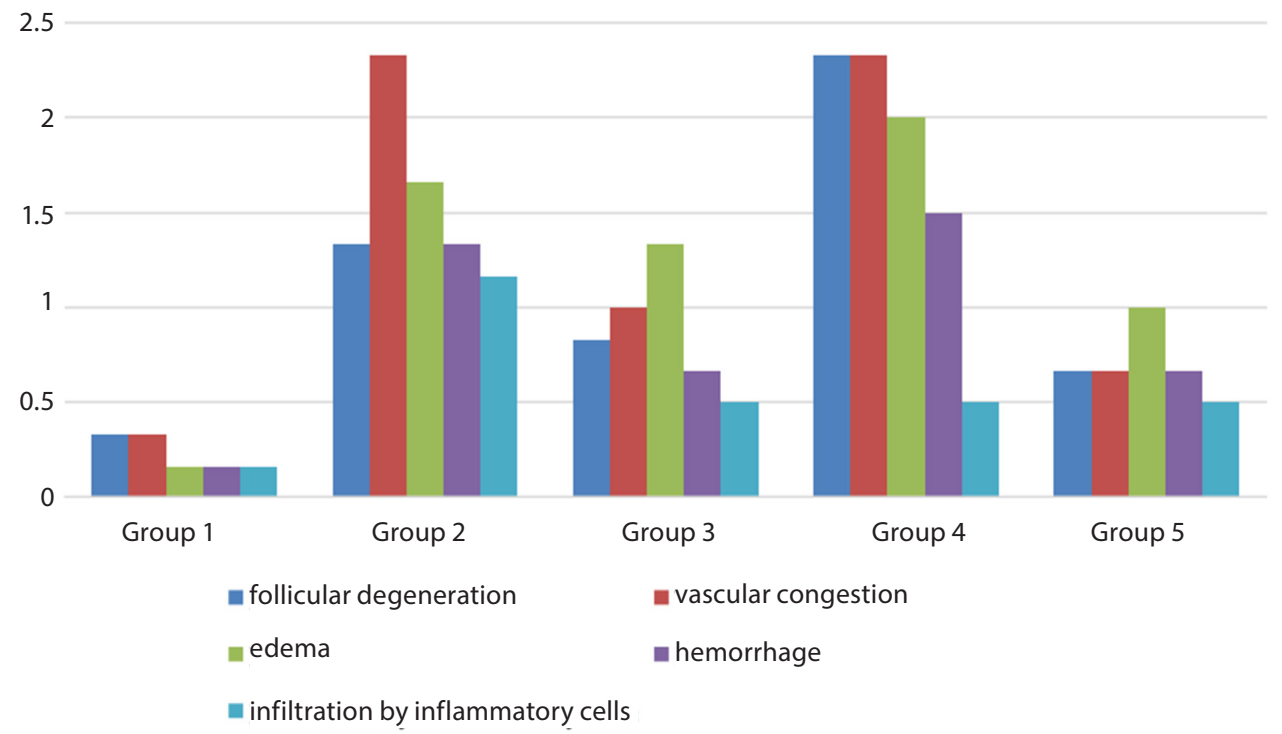

Fig. 2. Scores of histopathological parameters by group

\section{Discussion}

Ovarian torsion is a true gynecological emergency. Its prevalence is about $3 \%$ and this is rising due to the use of infertility treatments. ${ }^{1,2}$ Difficulties in diagnosis prolong the time between the onset of symptoms and treatment. Once a diagnosis of ovarian torsion is confirmed, immediate intervention is necessary. Conservative treatment of the ovary should be the first choice regardless of the tissue color.

After de-torsion of the twisted ovary, the tissue will again receive blood supply and this could trigger further damage, known as ischemia/reperfusion injury. Once the blood flow is disrupted, a series of events lead to tissue injury and cell death. These processes are largely related to energy production and utilization. Reduced energy decreases intracellular ATP levels. Ischemia induces anaerobic energy utilization, which causes an accumulation of the products of anaerobic metabolism and reduces intracellular $\mathrm{pH} .{ }^{5}$ These processes are reversible if the blood supply is reestablished. However, reperfusion of the tissue is not a situation one should ignore. The reintroduction of oxygen and energy into an ischemic cellular environment 


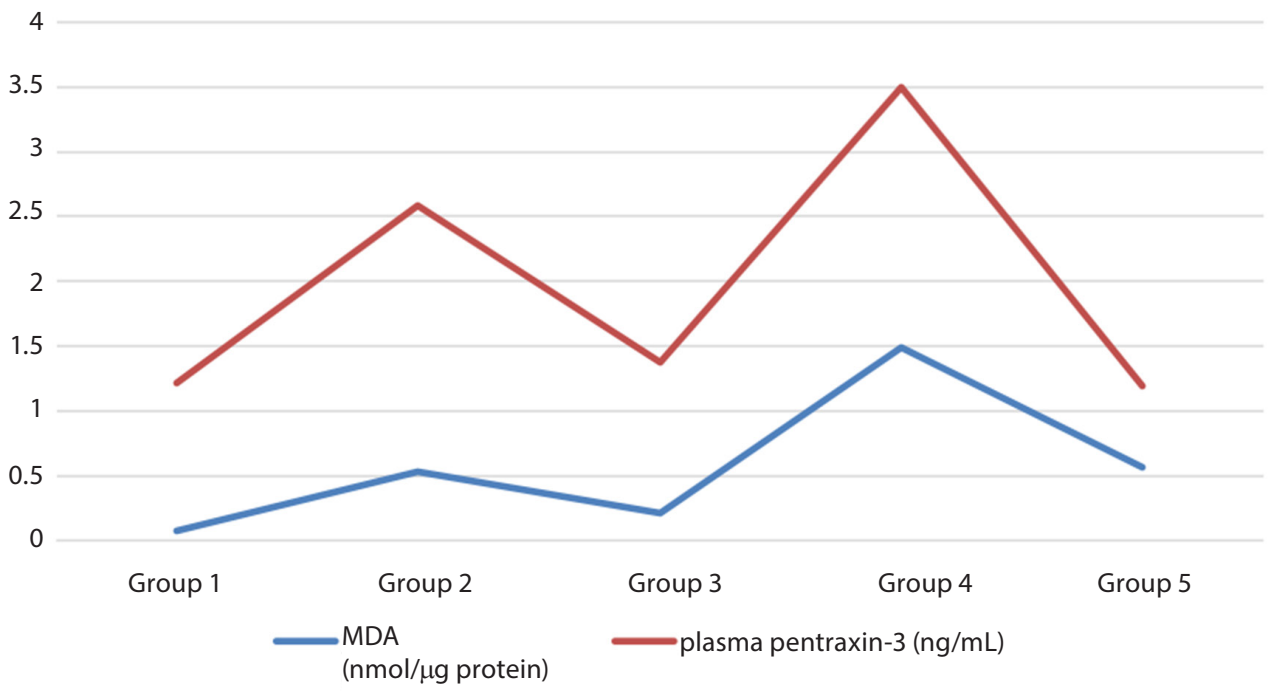

Fig. 3. Tissue MDA and plasma pentraxin-3 levels by group

Table 1. Histopathological parameters by group

\begin{tabular}{|l|c|c|c|c|c|}
\hline Histopathological parameters & $\begin{array}{c}\text { Group 1 } \\
\text { - control } \\
\text { (sham-operated) }\end{array}$ & $\begin{array}{c}\text { Group 2 } \\
\text { - torsion group }\end{array}$ & $\begin{array}{c}\text { Group 3 } \\
\text { - torsion } \\
+ \text { GB group }\end{array}$ & $\begin{array}{c}\text { Group 4 } \\
\text { - torsion/de-torsion } \\
\text { group }\end{array}$ & $\begin{array}{c}\text { Group 5 } \\
\text { - torsion/de-torsion } \\
+ \text { GB group }\end{array}$ \\
\hline Follicular degeneration & $0.33 \pm 0.21$ & $1.33 \pm 0.21^{*}$ & $0.83 \pm 0.16^{\#}$ & $2.33 \pm 0.33$ & $0.66 \pm 0.21^{+\dagger}$ \\
\hline Vascular congestion & $0.33 \pm 0.21$ & $2.33 \pm 0.21^{* *}$ & $1.0 \pm 0.25^{\#}$ & $2.33 \pm 0.33$ & $0.66 \pm 0.21^{+\dagger}$ \\
\hline Edema & $0.16 \pm 0.16$ & $1.66 \pm 0.33^{*}$ & $1.33 \pm 0.42^{\text {a }}$ & $2.0 \pm 0.36$ & $1.0 \pm 0.25^{\dagger}$ \\
\hline Hemorrhage & $0.16 \pm 0.16$ & $1.33 \pm 0.21^{*}$ & $0.66 \pm 0.21^{\#}$ & $1.5 \pm 0.22$ & $0.66 \pm 0.21^{\dagger}$ \\
\hline Infiltration by inflammatory cells & $0.16 \pm 0.16$ & $1.16 \pm 0.16^{*}$ & $0.5 \pm 0.22^{\#}$ & $1.33 \pm 0.33$ & $0.5 \pm 0.22^{\dagger}$ \\
\hline
\end{tabular}

${ }^{*} p<0.01$, Group 2 vs Group 1; ** $p<0.0001$, Group 2 vs Group 1; ${ }^{\#} p<0.05$, Group 3 vs Group 2; ${ }^{a}$ p $>0.05$, Group 3 vs Group 2; ${ }^{\dagger}$ p $<0.05$, Group 5 vs Group 4; ${ }^{++} \mathrm{p}<0.01$, Group 5 vs Group 4.

Table 2. Tissue MDA and plasma pentraxin-3 levels by group

\begin{tabular}{|c|c|c|c|c|c|}
\hline Tissue levels & $\begin{array}{l}\text { Group } 1 \\
\text { control (sham- } \\
\text { operated) }\end{array}$ & $\begin{array}{c}\text { Group } 2 \\
\text { - torsion group }\end{array}$ & $\begin{array}{c}\text { Group } 3 \\
\text { torsion + GB group }\end{array}$ & $\begin{array}{c}\text { Group } 4 \\
\text { torsion/de-torsion } \\
\text { group }\end{array}$ & $\begin{array}{c}\text { Group } 5 \\
\text { torsion/de-torsion } \\
+ \text { GB group }\end{array}$ \\
\hline MDA [nmol/ug protein] & $0.07 \pm 0.009$ & $0.53 \pm 0.16^{*}$ & $0.21 \pm 0.04^{\#}$ & $1.49 \pm 0.18$ & $0.56 \pm 0.10^{+}$ \\
\hline Plasma pentraxin-3 [ng/mL] & $1.21 \pm 0.20$ & $2.58 \pm 0.14^{*}$ & $1.38 \pm 0.29^{\#}$ & $3.5 \pm 0.36$ & $1.19 \pm 0.18^{+}$ \\
\hline
\end{tabular}

MDA - malondialdehyde; * $p<0.05$, Group 2 vs Group 1; ${ }^{*} p<0.05$, Group 3 vs Group 2; ${ }^{\dagger} p<0.001$, Group 5 vs Group 4.

can trigger cell injury by damaging cellular and organelle membranes, including the mitochondria, by free radical formation, by the aggregation of leukocytes and inflammatory mediators, and by the activation of the pro-apoptotic signaling cascade, and complement systems. ${ }^{5,18,19}$ All these processes are called ischemia/reperfusion injury.

The primary treatment of ovarian torsion is de-torsion of the twisted ovary. However, due to the aforementioned reperfusion damage, concomitant treatment modalities with surgery are obviously required to reduce injury to the ovarian tissue. In our study, we investigated the effects of GB in I/R injury. Over 400 clinical trials have been performed, looking at a variety of medicinal properties and clinical uses to prevent or contribute beneficial effects for the regression of several diseases. ${ }^{20}$ Ginkgo biloba extracts, such as terpene lactones and ginkgo flavone glycosides, are purported to be responsible for the beneficial effects. ${ }^{21}$ Terpene lactones not only inhibit the platelet-activating factor due to selective antagonism, but also act as selective use-dependent blockers of glycine-activated chloride channels in the central nervous system. ${ }^{22}$ In another study, the authors studied GB leaves with high-performance liquid chromatography and indicated that flavonoids in GB had powerful antioxidant attributes. ${ }^{23}$

Gingko biloba bilobalide plays a crucial role in mitochondrial protection during ischemia. Bilobalide allows the mitochondria to maintain respiratory activity at decreased oxygen levels, which delays ischemia-induced injury. ${ }^{24}$ In our study, we also aimed to investigate GB's protector role for ischemic injury. We found that the GB torsion group had lower histopathological scores than the torsion-only group. Flavonoids also have antioxidative and 
radical scavenger effects. They decrease reactive oxygen species and inhibit membrane lipid oxidation due to the scavenging of superoxide anions, hydroxyl radicals, and peroxyl radicals, and they prevent lipid peroxidation. ${ }^{25}$ Flavonoids also act as suppressors of the immune system and reduce inflammatory cytokine production, especially TNF- $\alpha$, IL-1 $\beta$, and prostaglandin E2. ${ }^{26}$ Our study is the only one in the literature that has investigated the effect of $\mathrm{GB}$ in $\mathrm{I} / \mathrm{R}$ injury in ovarian tissue. We have reported that GB certainly decreased I/R as well as ischemic injury with improved histopathological parameters and tissue and plasma oxidative stress markers in a rat model. The rats which were administered GB had less follicular degeneration, vascular congestion, edema, hemorrhage, and infiltration by inflammatory cells compared with the rats which were not administered GB.

Ginkgo biloba has been widely used in several studies. The proper dosage of GB to initiate I/R injury protection has not been described in the literature. In one study, no significant adverse effects occurred in patients taking $600 \mathrm{mg}$ of leaf extracts in single doses. ${ }^{27}$ In experimental animal studies, the dosage of GB has varied between $15 \mathrm{mg} / \mathrm{kg}$ and $400 \mathrm{mg} / \mathrm{kg}^{27-29} \mathrm{We}$ administered $80 \mathrm{mg} / \mathrm{kg}$ of $\mathrm{GB}$ for each rat in order to evaluate its effects on $\mathrm{I} / \mathrm{R}$ injury. Although no significant adverse effects occurred in patients taking $600 \mathrm{mg}$ of leaf extracts in single doses, GB is not definitely a safe herbal medicine, as mentioned in one review, because it was reported that it could cause epileptic seizures, unconsciousness, or paralysis of the legs, and may even cause death because of ginkgotoxin, which can be found especially in the GB seeds. ${ }^{30}$

Tissue and plasma oxidative stress markers were also compared between groups. There are several oxidative stress markers, such as total antioxidant status, malondialdehyde, myeloperoxidase, and 8-hydroxy dehydrogenase, which can be investigated in tissue samples to evaluate the tissue I/R injury. Malondialdehyde is one of the most investigated markers in I/R injury. In several recent studies, MDA was used to evaluate tissue injury induced by I/R oxidative stress. ${ }^{8-10}$ In our study, the elevated MDA levels in the torsion and torsion/de-torsion groups were consistent with the literature. Furthermore, the GB rats had significantly lower MDA levels. These results demonstrate that GB could be a beneficial supplementary medicine for the prevention of $I / R$ injury if our study is supported by further clinical studies carried out on human beings.

In our study we used pentraxin-3 to evaluate the inflammatory process in rats. PTX3 is a glycoprotein that belongs to the PTX family of acute-phase proteins, such as C-reactive protein (CRP) and serum amyloid P component (SAP). It has been widely studied in the diagnosis of ischemic heart disease. ${ }^{7,31,32}$ In a recent experimental rat study, Akman et al. stated that PTX3 could be a specific acute-phase reactant detected in ovarian injuries such as ovarian torsion, and that it could be used as a diagnostic marker. ${ }^{33}$ In this study, plasma PTX3 was significantly increased in the torsion and torsion/de-torsion groups. To our knowledge, PTX3 could be a good marker for ovarian injury. We also found that GB relieved I/R injury because of the lower values in the GB groups in comparison with the non-GB groups.

In conclusion, our study is the first and only study that investigates GB on ovarian I/R injury in a rat model. At present, GB is already being used as a dietary supplement, but could become a treatment modality for ischemia/ reperfusion injury along with de-torsion surgery if our results are also supported by further clinical studies. It could be a useful dietary supplement or may become a new medicine in the future which can also relieve ischemic injury. Clinical studies with larger sample sizes are needed for more valuable decisions.

\section{References}

1. Hibbard LT. Adnexal torsion. Am JObstet Gynecol. 1985;152(4):456-461.

2. Mashiach S, Bider D, Moran O, Goldenberg M, Ben-Rafael Z. Adnexal torsion of hyperstimulated ovaries in pregnancies after gonadotropin therapy. Fertil Steril. 1990;53(1):76-80.

3. Houry D, Abbott JT. Ovarian torsion: A fifteen-year review. Ann Emerg Med. 2001;38(2):156-159.

4. Huchon C, Fauconnier A. Adnexal torsion: A literature review. Eur J Obstet Gynecol Reprod Biol. 2010;150(1):8-12.

5. Jennings RB, Murry CE, Steenbergen C Jr, Reimer KA. Development of cell injury in sustained acute ischemia. Circulation. 1990;82(3):II2-12.

6. Diamond JM, Wigfield CH. Role of innate immunity in primary graft dysfunction after lung transplantation. Curr Opin Organ Transplant. 2013;18(5):518-523.

7. Zhu H, Cui D, Liu K, et al. Long pentraxin PTX3 attenuates ischemia reperfusion injury in a cardiac transplantation model. Transpl Int. 2014;27(1):87-95.

8. Ergenoglu M, Erbaş O, Akdemir A, et al. Attenuation of ischemia/ reperfusion-induced ovarian damage in rats: Does edaravone offer protection? Eur Surg Res. 2013;51(1-2):21-32.

9. Yildirim N, Yigitturk G, Sahingoz Yildirim AG, et al. Octreotide protects ovary against ischemia-reperfusion injury in rats: Evaluation of histological and biochemical parameters. J Obstet Gynaecol Res. 2015;41(10):1591-1597.

10. Akdemir A, Erbas O, Gode F, et al. Protective effect of oxytocin on ovarian ischemia-reperfusion injury in rats. Peptides. 2014;55:126-130.

11. Wada K, Ishigaki S, Ueda K, et al. Studies on the constitution of edible and medicinal plants. I. Isolation and identification of 4-O-methylpyridoxine, toxic principle from the seed of Ginkgo biloba L. Chem Pharm Bull. 1988;36(5):1779-1782.

12. Oyama Y, Fuchs PA, Katayama N, Noda K. Myricetin and quercetin, the flavonoid constituents of Ginkgo biloba extract, greatly reduce oxidative metabolism in both resting and $\mathrm{Ca}(2+)$-loaded brain neurons. Brain Res. 1994;635(1-2):125-129.

13. Benzi G, Moretti A. Are reactive oxygen species involved in Alzheimer's disease? Neurobiol Aging. 1995;16(4):661-674.

14. Guo RZ, Liu XG, Gao W, et al. A strategy for screening antioxidants in Ginkgo biloba extract by comprehensive two-dimensional ultra high performance liquid chromatography. J Chromatogr A. 2015; 27(1422):147-154

15. Guven S, Muci E, Unsal MA, et al. The effects of carbon dioxide pneumoperitoneum on ovarian blood flow, oxidative stress markers, and morphology during laparoscopy: A rabbit model. Fertil Steril. 2010;93(4):1327-1332.

16. Tukozkan N, Erdamar H, Seven I. Measurement of total malondialdehyde in plasma and tissues by high-performance liquid chromatography and thiobarbituric acid assay. Firat Medical Journal. 2006;11(2): 88-92.

17. Bradford MM. A rapid and sensitive method for the quantitation of microgram quantities of protein utilizing the principle of proteindye binding. Anal Biochem. 1976;72:248-254. 
18. Saraste A, Pulkki K, Kallajoki M, et al. Apoptosis in human acute myocardial infarction. Circulation. 1997;95(2):320-323.

19. Cannon RO $3^{\text {rd }}$. Mechanisms, management and future directions for reperfusion injury after acute myocardial infarction. Nat Clin Pract Cardiovasc Med. 2005;2(2):88-94.

20. Ude C, Schubert-Zsilavecz M, Wurglics M. Ginkgo biloba extracts: A review of the pharmacokinetics of the active ingredients. Clin Pharmacokinet. 2013;52(9):727-749.

21. Isah T. Rethinking Ginkgo biloba L. Medicinal uses and conservation Pharmacogn Rev. 2015;9(18):140-148.

22. Nakanishi K. Terpene trilactones from Ginkgo biloba: From ancient times to the $21^{\text {st }}$ century. Bioorg Med Chem. 2005;13(17):4987-5000.

23. Ding XP, Qi J, Chang YX, et al. Quality control of flavonoids in Ginkgo biloba leaves by high-performance liquid chromatography with diode array detection and on-line radical scavenging activity detection. J Chromatogr A. 2009:1216(11):2204-2210.

24. Janssens D, Michiels C, Delaive E, et al. Protection of hypoxiainduced ATP decrease in endothelial cells by Ginkgo biloba extract and bilobalide. Biochem Pharmacol. 1995;50(7):991-999.

25. Smith JV, Luo Y. Studies on molecular mechanisms of Ginkgo biloba extract. Appl Microbiol Biotechnol. 2004;64(4):465-472.
26. Zheng LT, Ock J, Kwon B, Suk K. Suppressive effects of flavonoid fisetin on lipopolysaccharide-induced microglial activation and neurotoxicity. Int Immunopharmacol. 2008;8(3):484-494.

27. Pizzorno JE, Murray MT. A Textbook of Natural Medicine. Seattle, WA: John Bastyr College Publications; 1985.

28. Das A, Shanker G, Nath C, et al. A comparative study in rodents of standardized extracts of Bacopa monniera and Ginkgo biloba: Anticholinesterase and cognitive enhancing activities. Pharmacol Biochem Behav. 2002;73(4):893-900.

29. Yallapragada PR, Velaga MK. Effect of Ginkgo biloba extract on leadinduced oxidative stress in different regions of rat brain. J Environ Pathol Toxicol Oncol. 2015;34(2):161-173.

30. Leistner E, Drewke C. Ginkgo biloba and ginkgotoxin. J Nat Prod. 2010;73(1):86-92.

31. Shimizu T, Suzuki S, Sato A, et al. Cardio-protective effects of pentraxin-3 produced from bone marrow-derived cells against ischemia/ reperfusion injury. J Mol Cell Cardiol. 2015;2828(15):30084-30085.

32. Hansson GK, Libby P. The immune response in atherosclerosis: A double-edged sword. Nat Rev Immunol. 2006;6(7):508-519.

33. Akman L, Erbas O, Terek MC, et al. The long pentraxin-3 is a useful marker for diagnosis of ovarian torsion: An experimental rat model. J Obstet Gynaecol. 2015;15:1-4. 